\title{
Commentary
}

\section{NIH can no longer turn its back on Chronic Fatigue Syndrome}

\author{
Arthur A. Mirin \\ Independent Researcher, Castro Valley, CA, USA \\ E-mail:aamirin@comcast.net
}

Received 28 September 2019

Accepted 22 January 2020

Myalgic Encephalomyelitis/Chronic Fatigue Syndrome (ME/CFS) is a debilitating disease with immune, neurological and energy metabolism abnormalities. It affects 1 to 2.5 million Americans and costs the U.S. economy $\$ 18-24 \mathrm{~B}$ a year in lost productivity and medical costs [1]. The disease has no definitive biomarkers, and there are no FDAapproved treatments specifically for ME/CFS.

In May 2019, the National Institutes of Health (NIH) announced that a team led by Dr. Ron Davis, Director of the Stanford Genome Technology Center at Stanford University, had developed a blood test that, in a pilot study, accurately differentiated people with ME/CFS from healthy controls via a difference in the electrical responses of blood cells to a salt stressor [2]. This news was particularly exciting because of the possibility of this leading to a definitive diagnostic test as well as the possibility of this technique being used to screen for potential treatments.

What NIH failed to advertise is that Open Medicine Foundation and Stanford University privately funded this work. While the NIH did fund the development of the underlying technology, they also cut the funding before the team could apply it to diseases such as ME/CFS, saying that it was not in the NIH's mission to reduce the cost of health care.

Low funding and negligible commitment to $\mathrm{ME} / \mathrm{CFS}$ research is nothing new. For over thirty years, ME/CFS was viewed as a psychogenic illness as opposed to being physiologically-based. This resulted in scant governmental research support, which drove researchers away from the field, and in turn depressed the level of government funding further, resulting in a continuing cycle of disinterest.

In 2015 the National Academy of Medicine (NAM) published a comprehensive report that provided evidence of neurological, immunological, autonomic and energy metabolism impairment in those with ME/CFS [1]. However, NIH has made only modest increases to an already inadequate research budget and shows no signs of enacting further increases.

In 2016 I co-authored a journal article [3] that estimated the disease burden (a measure combining morbidity and mortality) of ME/CFS in the United States. Results of applying that methodology to more recent data are presented in this special issue of Work [4]. We find that the ME/CFS burden is double that of HIV/AIDS and over half that of breast cancer [4].

Following an approach already adopted by NIH, we performed a statistical analysis to establish NIH funding levels commensurate with disease burden. We estimate that ME/CFS should be funded annually at slightly over $\$ 200 \mathrm{M}$, whereas it currently receives only $\$ 15 \mathrm{M}$ from the NIH [4]. In fact, among those diseases for which the NIH provides both funding and burden data, ME/CFS is the most underfunded, at 
roughly seven percent of that commensurate with its disease burden [4]. It has now been five years since the NAM recognized ME/CFS as "serious, chronic, complex and systemic". The fact that ME/CFS remains so underfunded is incomprehensible and inexcusable.

The NIH must substantially increase ME/CFS funding and encourage research proposals by issuing ME/CFS-specific funding opportunities with set-aside funds to overcome the lack of research applications. To its credit, the NIH did allocate additional funds to establish several Collaborative Research Centers in late 2017. But they rejected over twice as many Center applications as they funded, including those from world-class researchers. Even with the launch of these Centers, ME/CFS remains the lowest funded disease (burdenwise) among those diseases for which the NIH provides both funding and burden data.

The NIH claims that ME/CFS science is not ready for accelerated funding, and in particular, not enough is known yet to consider clinical trials. But at the April 2019 NIH-sponsored "Accelerating Research on ME/CFS" Conference, leading researchers echoed the sentiment that the science is indeed ready and that we must increase funding now [5]. Scientists working with the advocacy group MEAction have recently presented a detailed analysis of the inadequacies of NIH's urgent-lacking approach and a comprehensive set of recommendations as to how NIH should move forward [6]. Meanwhile, a plethora of researchers, many early-career and eager, are exploring promising avenues on the shoestring budgets of nonprofit organizations.

The NIH needs to underwrite the discovery necessary to hone in on a solution. The NIH cannot continue to shortchange ME/CFS - a disease more impactful than HIV/AIDS and almost as impactful as breast cancer - a disease with over one quarter home- bound or bedbound and between one- and two-thirds unemployed. Private investment cannot make up for what the NIH is not providing. The NIH has been receiving multi-billion dollar increases in its yearly budget [7]. The NIH needs to show a commitment that is commensurate with the burden and severity of this disease.

\section{Conflict of interest}

None to report.

\section{References}

[1] NAM - National Academy of Medicine, National Academy of Sciences. Beyond Myalgic Encephalomyelitis/Chronic Fatigue Syndrome: Redefining an Illness; 2015. Available from http://www.nationalacademies.org/hmd/Reports/2015/ ME-CFS.aspx

[2] National Institutes of Health; 2019. Available from https:// www.nih.gov/news-events/nih-research-matters/blood-testmay-detect-myalgic-encephalomyelitis/chronic-fatiguesyndrome.

[3] Dimmock M, Mirin AA, Jason LA. Estimating the disease burden of ME/CFS in the United States and its relation to research funding. Journal of Medicine and Therapeutics 2016; 1. Available from http://www.oatext.com/pdf/JMT-1102.pdf.

[4] Mirin AA, Dimmock M, Jason LA. Research Update: The Relation between ME/CFS Disease Burden and Research Funding in the United States. WORK (this issue); 2020.

[5] Tucker, M. 'Milestone' Meeting Highlights NIH Efforts to Combat ME/CFS; 2019. Available from https://www. medscape.com/viewarticle/911906?src=wnl_edit_tpal\&uac= 265282FG\&impID=1939789\&faf=1\#vp_1.

[6] MEAction; 2019. Available from https://www.meaction.net/ wp-content/uploads/2019/10/NANDS-Report-Response_ 10-22-19.pdf.

[7] Congressional Research Service. National Institutes of Health (NIH) Funding: FY1994-FY2020. Updated April 4, 2019. Available from https://fas.org/sgp/crs/misc/R433 41.pdf. 